\title{
Esthetic Correction of Gummy Smile by Gingivectomy using Diode Laser
}

\author{
${ }^{1}$ Shivaprasad BM, ${ }^{2}$ MP Rakesh, ${ }^{3}$ Sandeep S Prabhu
}

\begin{abstract}
A pleasant smile is considered a symbol of beauty and well being in the modern society periodontology extensively deals with soft tissue surgeries. However, pain, trauma, bleeding, postoperative edema and scarring are often associated with conventional methods of surgery which are problematic for both the patient and the clinician. Hence, to overcome these drawbacks, $940 \mathrm{~nm}$ diode laser was used to perform various periodontal surgical procedures. This case report highlights the use of diode laser to correct gummy smile for better shape, contour and gingival margin.
\end{abstract}

Keywords: Diode laser, Excessive gingival display, Gingivectomy, Gummy smile.

How to cite this article: Shivaprasad BM, Rakesh MP, Prabhu SS. Esthetic Correction of Gummy Smile by Gingivectomy using Diode Laser. J Health Sci Res 2015;6(1):17-21.

Source of support: Nil

Conflict of interest: None

\section{INTRODUCTION}

Beauty lies in the eyes of the beholder. Keeping this old adage in mind, smile corrections can be done in patients with cosmetic concerns. However, a pleasant smile varies from person to person, but usually depends on the extent of gingival exposure. When a person smiles, the entire crowns of maxillary central incisors and $1 \mathrm{~mm}$ of pink attached gingiva is visible. An exposed gingiva of $2-3 \mathrm{~mm}$ is cosmetically acceptable. A gummy smile is seen usually when more than $3 \mathrm{~mm}$ of gingiva is visible. The form of the lips and the position of lips during speech and smiling cannot be changed easily, but the dentist can modify/control the form of the teeth, interdental papilla, and position of the gingival margin and incisal edges of the teeth. While correcting the position of the marginal gingiva, one should be cautious in preserving the biologic width. Often, osseous recontouring becomes

\footnotetext{
${ }^{1,3}$ Reader, ${ }^{2}$ Postgraduate Student

${ }^{1-3}$ Department of Periodontology, Rajarajeswari Dental College and Hospital, Bengaluru, Karnataka, India
}

Corresponding Author: Shivaprasad BM, Reader, Department of Periodontology, Rajarajeswari Dental College and Hospital Bengaluru, Karnataka, India, Phone: 9901759011, e-mail: drspbmath@gmail.com essential to maintain the integrity of the biologic width and enable proper margin placement. Various methods have been documented including gingivectomy, flap surgery with osseous contouring, apically displaced flaps and orthodontic therapy. Excessive display of the gingiva is sometimes caused by vertical maxillary excess and a long mid-face. ${ }^{1}$

When an excess of gingiva superior to the maxillary anterior teeth is displayed upon full smile, it is termed a gingival smile. The gingival smile is known by a variety of terms including gummy smile, high lip line, short upper lip, and full denture smile. Perhaps this variety in terms is indicative of the many different causes of a gummy smile. ${ }^{2}$

The smile itself and the esthetics of the smile are influenced by three components: teeth, gums and lips. An attractive smile depends on the proper proportion and arrangement of these three elements.

The upper lip should symmetrically expose up to $3 \mathrm{~mm}$ of the gum and the gum line must follow the contour of the upper lip. The exposure of more than $3 \mathrm{~mm}$ of the gum during the smile is known as gingival or gummy smile. For some patients, gummy smile represents an esthetic disorder. Hulsey noted that the most attractive smiles were those in which the upper lip rested at the height of the gingival margin of the maxillary incisor. ${ }^{2}$

Gummy smiles range from mild, moderate, and advanced, to severe. Mazzuco classified gummy smile into anterior, posterior, mixed, or asymmetric, based on the excessive contraction of muscles involved. ${ }^{3}$

Goldstein classified the smile line (consisting of the lower edge of the upper lip during the smile) according to the degree of exposure of the teeth and gums into three types: high, medium, or low. ${ }^{4}$

Surgical reduction of the overgrown tissues is frequently necessary to accomplish an esthetic and functional outcome. The treatment consists of scalpel gingivectomy or laser gingivectomy. Laser is one of the most promising new technical modalities in periodontal treatment. ${ }^{5}$

However, some laser wavelengths work on both hard and soft tissues (2,780 and 2,940 nm) while other lasers, such as the $810 \mathrm{~nm}$ diode work on only soft tissues and have a very good surgical and hemostatic action on soft tissues following maxillary vestibular frenectomies, 
gingivectomies and recontouring of gingival overgrowth, surgical exposure of buccally and palatally placed teeth and operculectomies. Also, the soft tissue diode lasers have an excellent incision performance with a cutting depth of 2-6 $\mathrm{mm}$ and have an added advantage over conventional surgery in that there is a sealing of small blood and lymphatic vessels resulting in hemostasis and reduced postoperative edema. Target tissues are also disinfected as a result of local heating and production of an eschar layer and a decreased amount of scarring due to decreased postoperative tissue shrinkage. Consequently, the use of sutures is eliminated. ${ }^{5}$

Previously, conventional surgical procedures were performed using a scalpel under local anesthesia for these procedures. However, the use of laser has become more popular with studies demonstrating the use of the argon $(514.5 \mathrm{~nm})$, carbon dioxide $\left(\mathrm{CO}_{2}, 10,600 \mathrm{~nm}\right)$, neodymium: yttrium-aluminum-perovskite (Nd:YAP, $1,340 \mathrm{~nm}$ ), neodymium-yttrium aluminium garnet (NdYAG, $1060 \mathrm{~nm}$ ), and gallium-aluminum-arsenide surgical diode (GaAlAs, $980 \mathrm{~nm}$ ) lasers. ${ }^{5}$

Hence, this case report was aimed at correction of excessive gingival display by gingivectomy using diode laser.

\section{CASE REPORT}

A female patient aged 23 years visited the Department of Periodontology, Rajarajeswari Dental College and Hospital, Bengaluru, with the chief complaint of gummy smile.

Pretreatment photographs were taken (Fig. 1). When the patient relaxed, we took another smiling image, which showed a significant gummy smile with more than $4 \mathrm{~mm}$ of gingival exposure in the incisor region. The photographs show bilateral class-I canine and molar relationships with a slightly deep overbite and overjet and moderate crowding in lower anterior.

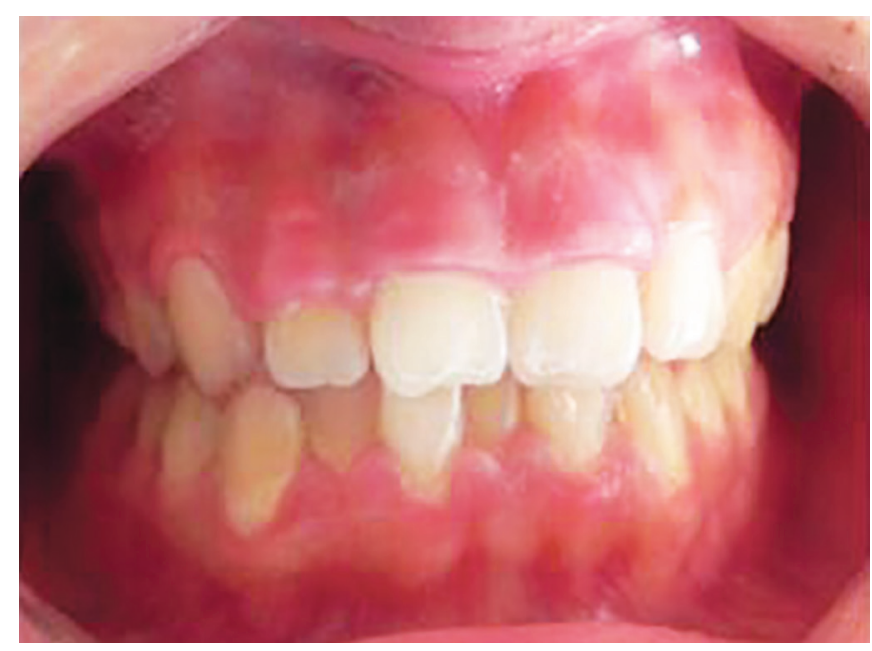

Fig. 1: Preoperative view showing excessive gingival display
The clinical examination suggested incisal and canine guidance without prematurity and shift. There was an excessive gingival display. The patient had no temporomandibular joint symptoms. No deviation and pain during the border movement of the mandible were discovered. Patient's medical history was noncontributory and there were no contraindications to surgical treatment. With an exaggerated smile, the patient's teeth were visible from maxillary right canine to maxillary left canine.

Patient was asked to rate his/her pain experience and allocate a score before visual analog scale 1 (VAS1); and after treatment (VAS2) on the VAS. The difference (VAS1)-(VAS2) would be regarded as an indicator of the pain assessment.

Pain was measured using the $\mathrm{VAS}^{6}$ which is a horizontal line $10 \mathrm{~cm}$ in length anchored by word descriptors at each point and classified as follows:

Score $0-1 \mathrm{~cm}-$ no pain or distress

Score 2-3 cm-annoying

Score $4-5 \mathrm{~cm}$ - uncomfortable

Score 6-7 $\mathrm{cm}$-dreadful

Score 8-9 cm-horrible

Score $10 \mathrm{~cm}$-agonizing or unbearable distress.

Degree of gingival overgrowth as given by Bokenkamp et al: ${ }^{7}$

Grade 0: No signs of gingival overgrowth.

Grade 1: Overgrowth confined to Interdental papilla.

Grade 2: Overgrowth involves papilla and marginal gingiva.

Grade 3: Overgrowth covers three quarters or more of the crown.

On examination, we found grade 2 gingival overgrowth according to Bokenkamp et al 1994 bone sounding done in order to determine excessive gingival display. Bone sounding determined that $4 \mathrm{~mm}$ of gum tissue could safely be removed.

\section{TREATMENT}

When the gingival overgrowth is minimal, scaling of teeth and homecare may be all that is required to maintain good oral health. As the excess tissue increases, appearance and function indicate need for surgical intervention. The patient initially underwent phase 1 periodontal therapy that comprised scaling and root planning and oral hygiene instructions. The conventional external bevel gingivectomy is the most efficacious method of removing large quantities of gingival tissue, particularly when there is pseudo pocket with no attachment loss. However, this case report emphasized the use of diode laser as an alternative method for the correction of excessive gingival display by gingivectomy. 
Informed consent was obtained from the patient after discussion of the procedures that will be carried out. Local anesthesia (Xylocaine 2\% with Epinephrine 1:80,000) was administered. Bleeding points were marked with the help of the Krane-Kaplan pocket marker and the points were joined to prepare a line of excision (Fig. 2). The patient was instructed to wear protective goggles before activation of laser. Gingivectomy was performed using diode laser delivered using fiber optic technology (SIROLaser ${ }^{\circledR}$, Sirona, Bensheim, Germany) at $980 \pm 10 \mathrm{~nm}$ wavelength, a frequency of $1 \mathrm{~Hz}$, and time of $0.5 \mathrm{~ms}-\mathrm{cw}$, with $400 \mathrm{~nm}$ fiber diameter and $3.0 \mathrm{~W}$ of potency. The fiber optic tip was used in contact mode to perform gingivectomy. The laser was activated and gingival tissue was removed in a sweeping stroke joining the bleeding points (Fig. 3). A high-volume suction device was used during the procedure. The patient was recalled on day 7 and 3 months for postoperative evaluation and it was found that healing was uneventful.

On VAS, the patient marked as 4 (uncomfortable) on day 1 , and between 1 and 0 on day 7 , thus denoting it as mild annoying pain and no pain (Fig. 4). No periodontal pack was placed, and there was no bleeding and discomfort immediately after the procedure. Gingival shape, contour, scalloping were improved after the procedure (Figs 5 and 6).

\section{DISCUSSION}

For many intraoral soft tissue surgical procedures, the laser is a viable alternative to the conventional techniques. The commercially available dental instruments have emission wavelengths ranging from 488-10,600 nm and are all non ionizing radiation. This is to avoid any mutations in the cellular deoxyribonucleic acid (DNA) components which ionizing radiations are known to cause. Different wavelengths have different absorption

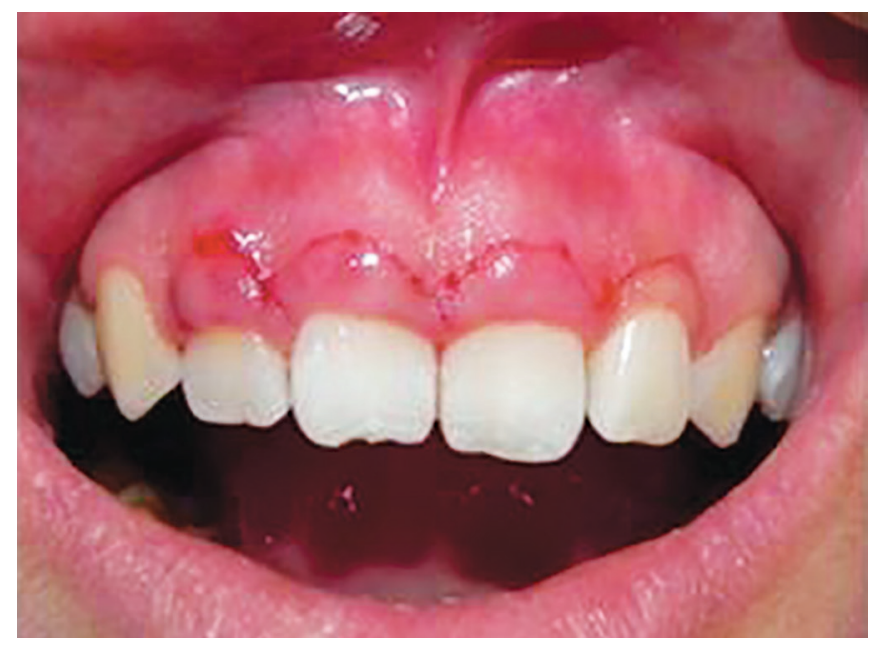

Fig. 2: Line of excision marked by joining the bleeding points made using Crane-Kaplan pocket marker coefficients based on the varied composition of human tissue. In order to maximize the thermal reaction, there should be a close match between the laser wavelength and the chromosphore(s) present in the target tissue. During the thermal ablation as the temperature increases at the surgical site, the soft tissues are subjected to warming $\left(37-60^{\circ} \mathrm{C}\right)$, protein denaturation, coagulation $\left(>60^{\circ} \mathrm{C}\right)$, welding $\left(70-90^{\circ} \mathrm{C}\right)$, vaporization $\left(100-150^{\circ} \mathrm{C}\right)$, and carbonization $\left(>200^{\circ} \mathrm{C}\right)$. The primary chromospheres for intraoral soft tissue ablation are hemoglobin, water, and melanin. ${ }^{8}$

Diode lasers (810-980 nm) emit laser light in the near infra-red spectrum of the electromagnetic radiation which are highly absorbed in hemoglobin and other pigments. One of the main benefits of using diode lasers is the ability to selectively and precisely interact with diseased tissues. Lasers also allow the clinician to reduce the amount of bacteria and other pathogens in the surgical field, and, in the case of soft-tissue procedures, achieve good hemostasis with no need for sutures. ${ }^{8,9}$

The purported advantages of lasers $v s$ conventional surgery include increased coagulation that yields a dry surgical field and better visualization; the ability to negotiate curvatures and folds within tissue contours; tissue surface sterilization and, therefore, reduction in bacteremia; decreased swelling, edema, and scarring; decreased pain; faster healing response; and increased patient acceptance. 9

When laser cutting is in progress, small blood and lymphatic vessels are sealed due to the generated heat, thereby reducing or eliminating bleeding and edema. Denatured proteins within tissue and plasma are the source of the layer termed 'coagulum', which is formed because of laser action and serves to protect the wound from bacterial or frictional action. Also the diode laser did not produce any deleterious effect on the root surface.

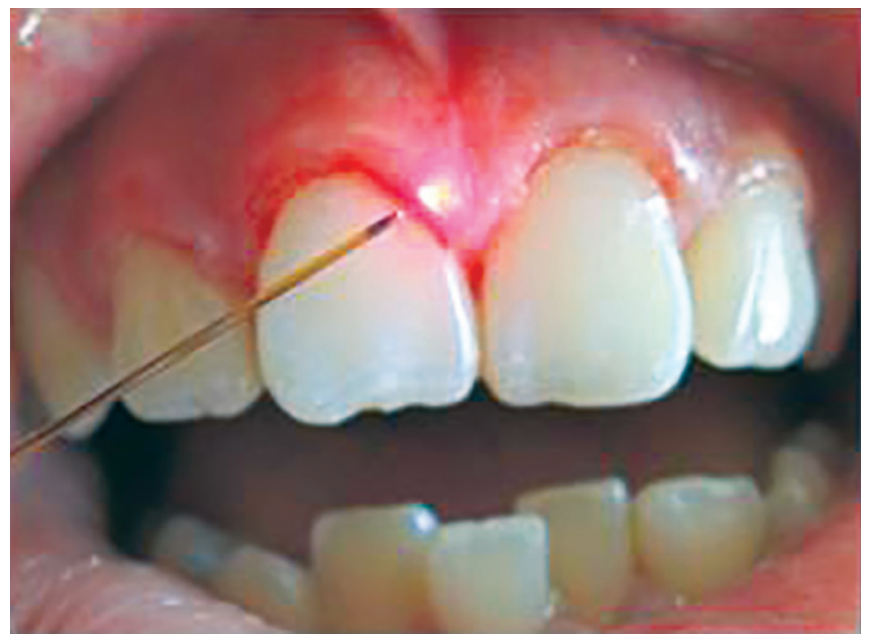

Fig. 3: Gingivectomy performed using diode laser 


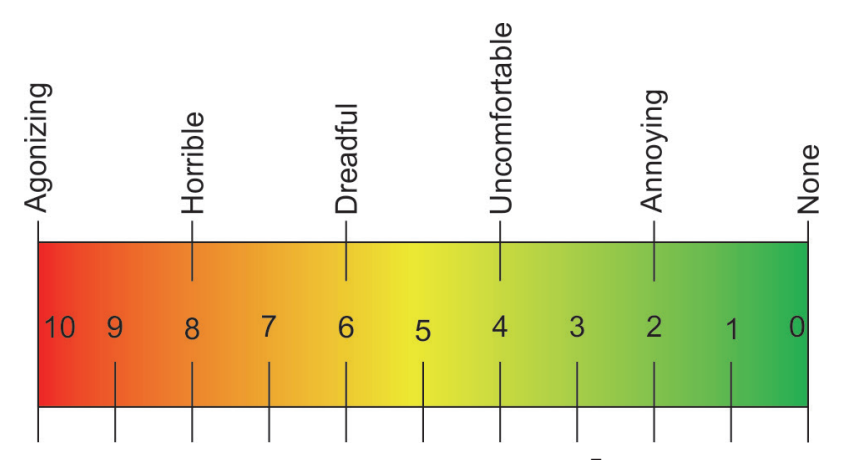

Fig. 4: Visual analog scale ${ }^{7}$

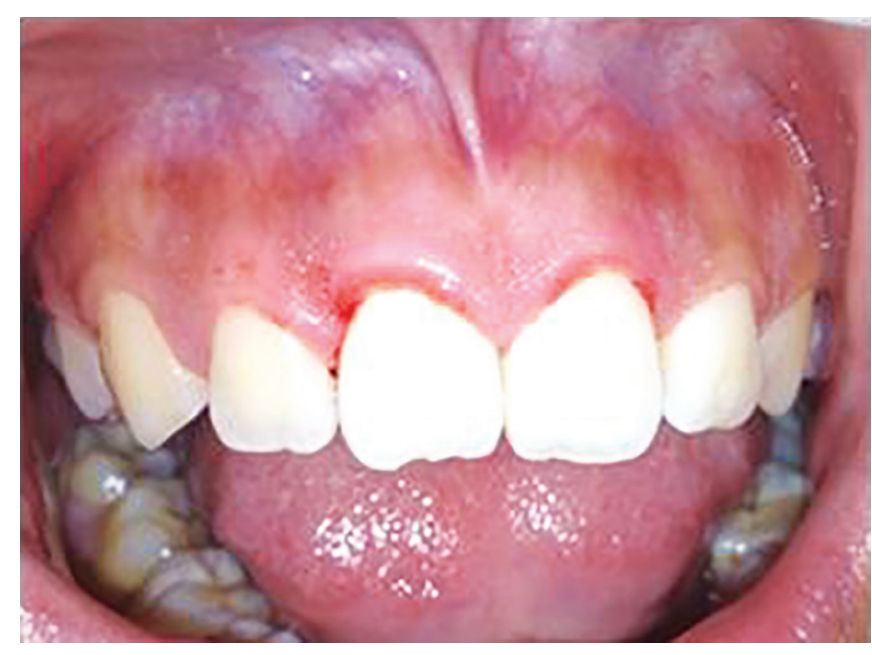

Fig. 5: One week postoperative photograph

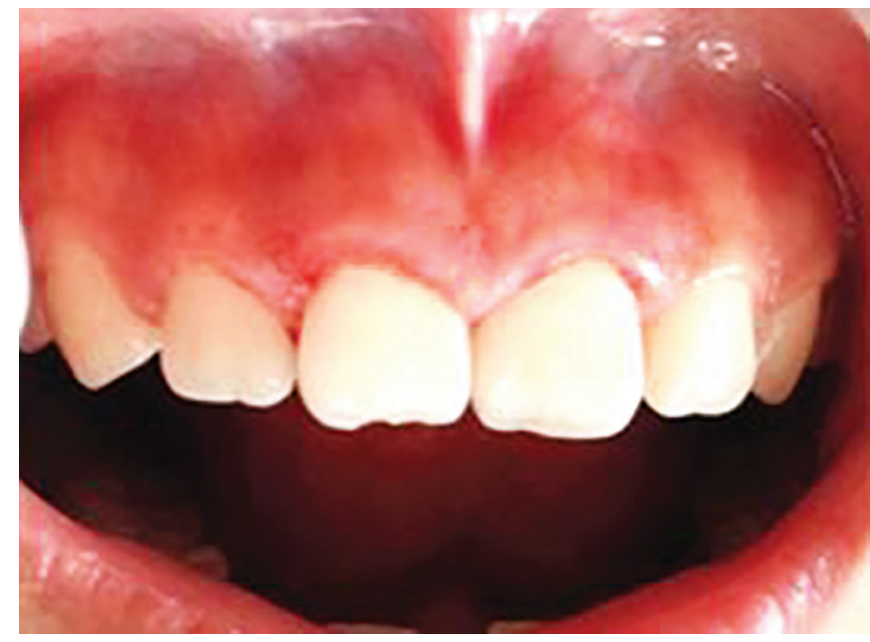

Fig. 6: Three months postoperative photograph

Therefore, diode laser surgery can be performed safely in close proximity to dental hard tissue. All these above mentioned advantages were evidently experienced in the above case. During procedure, there was no bleeding. Also, postoperatively, no pain was experienced by the patient and no swelling or any other signs of infection were noticed, whereas other alternative procedures have to be accompanied by administration of antibiotics and analgesics to minimize postoperative infection and pain..$^{10}$

Diode laser has a broad range of applications, which includes tissue retraction during restorative procedures, gingivectomy, gingivoplasty, crown-lengthening, frenectomy, bacterial decontamination, and removal of diseased epithelial lining during periodontal treatments. ${ }^{10}$

Another study reported that the use of low-level laser therapy (LLLT) following gingivectomy has a stimulatory effect and increases the fibroblast activity and collagen fiber formation. Laser therapy affects wound healing positively by enabling a more rapid epithelialization and by increasing revascularization. ${ }^{11}$

The diode laser is a useful instrument in oral soft tissue surgery and an alternative to conventional electro surgery and the use of scalpel. ${ }^{12}$ A stuxdy by Pirnat ${ }^{12}$ demonstrated the cutting ability of the soft tissue diode laser with a depth of $2-6 \mathrm{~mm}$ into the tissues with the sealing of small blood and lymphatic vessels as a result of the heat generated, thereby eliminating bleeding and edema.

However, another study ${ }^{13}$ indicated that scalpel repair was found to be equivalent or better than laser repair as a result of thermal damage to the tissues but also advocated the clinical use of the low level diode laser as an alternative to scalpel incision and suture repair.

Alternatives for gingival tissue removal include the use of a scalpel, electrosurgery, and/or lasers. The traditional surgical approach utilizing a scalpel blade exhibits the disadvantage of eliciting bleeding. Alternatively, electrosurgery has been utilized effectively to excise gingival tissue while simultaneously providing adequate hemostasis and is, therefore, preferred by many restorative dentists. Heat generation with this technique, however, occurs to a degree where an irreversible damage to the alveolar crest may result. ${ }^{14}$ Hence, Lasers offer the potential of increased operator control and minimal collateral tissue damage. Diode lasers, specifically, operate at a wavelength that is easily absorbed by the gingival tissues while posing a little risk of damaging the tooth structure.

The laser wound in the soft tissue has unique characteristics that are not found in any other surgically created wound. The result is a very superficial wound; it is not a burn, and the thermal damage caused by the irradiation is only a few tenths of a millimeter in depth. The cellular disintegration caused at the impact does not allow for the release of chemical mediators of inflammation, which leads to a reduced acute inflammatory response compared with scalpel created wounds. A thin layer of denatured collagen on the surface of the wound also reduces the degree of tissue irritation from oral fluids and serves as an impermeable dressing. Additionally, there is very little wound contraction. ${ }^{15}$

The prime rationale for the use of laser in this patient was to give a painless and bloodless substitute 
for the scalpel-facilitated surgical procedures. It also reduces the perception of fear and anxiety in the patient, thus instilling a positive attitude toward the dental treatment. Lasers also reduced the use of local anesthesia, periodontal dressing, and postoperative medications. It also reduces the effective chair side time, thus, leading to more cooperative behaviour of the patient.

Excessive gingival display (EGD) is a condition in which an overexposure of the maxillary gingiva ( $>3 \mathrm{~mm}$ ) is present during smiling. The proper diagnosis and determination of its etiology are essential for the selection of the right treatment modality. Different techniques have been used in cases of hyperactive upper lip: botulinum toxin injections, lip elongations with rhinoplasties, lip muscle detachments, myotomies, and lip repositions. This report presents a case of a young woman with an EGD larger than $4 \mathrm{~mm}$ during smiling caused by altered passive eruption, vertical maxillary excess, and a hyperactive upper lip that was treated with a modified lip repositioning technique and laser gingivectomy as she strongly refused orthognathic surgical treatment.

\section{CONCLUSION}

The diode laser represents the advanced treatment modality in oral healthcare. It is a surgical instrument that is reliable, portable, and powerful. It provides quick, precise soft tissue surgeries with minimal or no bleeding, swelling, or postoperative pain. This case report demonstrated that patient with excessive gingival display can be safely, easily, and effectively treated with minimal to no discomfort in the dental office by gingivectomy using diode laser resulting in better gingival shape, contour, scalloping and gingival margin were regained.

\section{REFERENCES}

1. Sarita N, Narayan TV, Jacob PC. Correction of gummy smile: a report of two cases. J Ind Soc Periodontol 2011;15(4):421-424.
2. Dolly PP, Sandip AT, Jaymin RS. Adjunctive treatment of gummy smile using botulinum toxin type: a case report. IOSR J Dent Med Sci 2012;3(2):22-29.

3. Mazzuco, Hexsel. Gummy smile and botulinum toxin: a new approach based on the gingival exposure area. J Am Acad Dermatol 2010;63(6):1042-1051.

4. Goldstein RE. Estética em Odontologia. Rio de Janeiro: Ed. Guanabara Koogan; 1980. p. 2-15.

5. Ize-iyamu INS, Saheeb BD, Edetanlen BE. Comparing the $810 \mathrm{~nm}$ diode laser with conventional surgery in orthodontic soft tissue procedures. Ghana Med J 2013;47(3): 107-111.

6. Hjermstad MJ, et al. Studies comparing numerical rating scales, verbal rating scales, and visual analog scales for assessment of pain intensity in adults: a systematic literature review. J Pain Symptom Manage 2011;41(6):1073-1093.

7. Bokenkamp A, Bohnhorst B, Beier C. Nifedipine aggravates cyclosporine: a induced hyperplasia. Pediatr Nephrol 1994;8(2):181-185.

8. Niemz MH. Laser-tissue interaction: fundamentals and applications. 3rd enlarged ed. Berlin, Germany: Springer; 2007. p. 45-46.

9. Goldman L. Chromophores in tissue for laser medicine and laser surgery. Lasers Med Sci 1990;5(3):289-292.

10. Govila V, Gulati M, Govila S. Diode laser applications in periodontics. Ind J Dental Sci 2011;5(3):64-67.

11. Firat ET, Dag A, Gunay A, et al. Histological examination of the effects of low-level laser therapy on healing of gingiva after gingivectomy in rats. Biotechnol Biotechnol Equip 2013; 27(5):4137-4140.

12. Pirnat S. Versatility of an $810 \mathrm{~nm}$ Diode laser in dentistry: an overview. Laha 2007;4(2):1-9.

13. D'Arcangelo C, Di Nardo Di Maio F, Prosperi GD Conte E, Baldi M, CaputiS. A preliminary study of healing of diodelaser versus scalpel incisions in rat oral tissue: a comparison of clinical, histopathological, and immunological results. Oral Surg Oral Med Oral Pathol Oral Radiol Endod 2007;103(6): 764-773.

14. Kalkwarf KL, Krejci RF, Edison AR, Reinhardt RA. Lateral heat production secondary to electrosurgical incisions. Oral Surg Oral Med Oral Pathol 1983;55(4):344-348.

15. Rossmann JA, Cobb CM. Lasers in periodontal therapy. Periodontol 2000 1995;9:150-164. 